\title{
The method of computer reconstruction of the ecological structure of intestinal microbiota communities based on metatranscriptome data
}

\author{
Kropochev A. ${ }^{1,2 *}$, Lashin S.A. ${ }^{1,2,3}$, Klimenko A.I. ${ }^{1,2}$ \\ ${ }^{1}$ Institute of Cytology and Genetics, SB RAS, Novosibirsk, Russia \\ ${ }^{2}$ Kurchatov Genomic Center of the Institute of Cytology and Genetics, SB RAS, Novosibirsk, Russia \\ ${ }^{3}$ Novosibirsk State University, Novosibirsk, Russia \\ *kropochev@bionet.nsc.ru
}

Key words: trait-based ecology, ecological networks, synthetic microbial communities, functional metagenomics

\begin{abstract}
Motivation and Aim: The human gut is a habitat for a complex microbial ecosystem of trillions of bacteria. It has been shown that certain changes in the taxonomic structure of the microbial community can be associated with the pathogenesis of numerous diseases [1]. However, since natural microbial communities consist of a large number of species, where different taxonomic groups can perform similar functions, it is rather difficult to identify ecological links between different bacteria. These problems force researchers to pay attention to trait-based approaches. Such approaches can reduce the complexity of system analysis by shifting the focus from taxonomy to ecological function.
\end{abstract}

Methods and Algorithms: We used publicly available data on a synthetic microbial community of 14 species, which served as a model for the human gut microbiota [2]. The developed pipeline includes the use of the Trinity platform [3] and the Kallisto program [4], as well as scripts written in the Python programming language.

Results: We have developed the method for computer reconstruction of the ecological structure of microbial communities. As part of its implementation, we carried out an expert reconstruction of the ecological structure of the microbial community, highlighting the following functional groups: butyrate-producers, mucin-decomposing bacteria, acetogens and sulfate-reducers. For each functional group, trait-determining genetic features were identified that were used to assess the activity of functional groups in the community based on metatranscriptome data. Subsequently, we verified the developed method by comparing changes in the activity of functional groups with changes in taxonomic abundance assessed using 16S rRNA sequencing data for a community under different conditions.

Conclusion: Further application of this method for the analysis of the ecological structure of natural microbial communities is of great interest.

\section{References}

1. Nicholson J.K. et al. Host-Gut Microbiota Metabolic interactions. Science. 2012;336(6086):12621267.

2. Desai M.S. et al. A dietary fiber-deprived gut microbiota degrades the colonic mucus barrier and enhances pathogen susceptibility. Cell. 2016;167(5):1339-1353.

3. Haas B.J. et al. De novo transcript sequence reconstruction from RNA-seq using the Trinity platform for reference generation and analysis. Nat. Protoc. 2013;8:1494-1512.

4. Bray N.L., Pimentel H., Melsted P., Pachter L. Near-optimal probabilistic RNA-seq quantification. Nat. Biotechnol. 2016;34:525-527. 